\title{
Asymptotic Behavior of SU(3) Toda System in a Bounded Domain
}

\author{
Chang-Shou Lin*, Juncheng Wei†, Chunyi Zhao ${ }^{\ddagger}$ \\ January 27, 2011
}

\begin{abstract}
We analyze the asymptotic behavior of blowing up solutions for the $\mathrm{SU}(3)$ Toda system in a bounded domain. We prove that there is no boundary blow-up point, and that the blow-up set can be localized by the Green function.
\end{abstract}

\section{Introduction}

In this paper, we consider the following SU(3) Toda system

$$
\begin{cases}\Delta u_{1 k}+2 \rho_{1 k} \frac{h_{1 k} e^{u_{1 k}}}{\int_{\Omega} h_{1 k} e^{u_{1 k}}}-\rho_{2 k} \frac{h_{2 k} e^{u_{2 k}}}{\int_{\Omega} h_{2 k} e^{u_{2 k}}}=0 & \text { in } \Omega, \\ \Delta u_{2 k}-\rho_{1 k} \frac{h_{1 k} e^{u_{1 k}}}{\int_{\Omega} h_{1 k} e^{u_{1 k}}}+2 \rho_{2 k} \frac{h_{2 k} e^{u_{2 k}}}{\int_{\Omega} h_{2 k} e^{u_{2 k}}}=0 & \text { in } \Omega, \\ u_{1 k}=u_{2 k}=0 & \text { on } \partial \Omega,\end{cases}
$$

where $\Omega \subset \mathbb{R}^{2}$ is a bounded domain, $\partial \Omega$ is its boundary and $\Delta$ is the Euclidean Laplacian. Here, $\rho_{1 k}$ and $\rho_{2 k}$ are two positive constants, $h_{1 k}(x)$ and $h_{2 k}(x)$ are two positive functions converging to $h_{1}(x)$ and $h_{2}(x)$ respectively in $C^{2, \beta}(\bar{\Omega})$ as $k \rightarrow \infty$. We are concerned with the asymptotic behavior of unbounded sequences of solutions to (1).

*Taida Institute of Mathematical Sciences, National Taiwan University, Taipei, 10617, Taiwan. Email: cslin@math.ntu.edu.tw

${ }^{\dagger}$ Department of Mathematics, The Chinese University of Hong Kong, Shatin, Hong Kong, China. Email: wei@math.cuhk.edu.hk

${ }^{\ddagger}$ Department of Mathematics, East China Normal University, Shanghai, 200241, China. Email: cyzhao@math.ecnu.edu.cn 
Toda system arises in many physical models. In particular, the Toda system (1) arises in the study of the non-Abelian non-relativistic ChernSimons theory with gauge group SU(3). See, for instance, the books [9], [25] and the references therein.

The analogous second order single mean-field equation

$$
\begin{cases}-\Delta u_{k}=\rho_{k} \frac{h_{k} e^{u_{k}}}{\int_{\Omega} h_{k} e^{u_{k}}} & \text { in } \Omega \subset \mathbb{R}^{2} \\ u_{k}=0 & \text { on } \partial \Omega\end{cases}
$$

has been extensively studied by many authors. We summarize some known results. Let $\left(u_{k}, \rho_{k}\right)$ be a blow-up sequence of solutions to $(2)$ with $\rho_{k}$ uniformly bounded, then it was proved that

(P1) (no boundary bubbles) $u_{k}$ is uniformly bounded near a neighborhood of $\partial \Omega$ (Nagasaki-Suzuki [19], Ma-Wei [18]);

(P2) (bubbles are simple) $\rho_{k} \rightarrow 8 m \pi$ for some integer $m \geq 1$ and (after taking a subsequence)

$$
u_{k}(x) \rightarrow 8 \pi \sum_{j=1}^{m} G\left(\cdot, x_{j}\right) \quad \text { in } C_{\mathrm{loc}}^{2}\left(\Omega \backslash\left\{x_{1}, \ldots, x_{m}\right\}\right),
$$

where $G$ is the Green function of $-\Delta$ with Dirichlet boundary condition. Furthermore, it holds that

$$
\nabla \ln h\left(x_{j}\right)+\nabla_{x} H\left(x_{j}, x_{j}\right)+\sum_{i \neq j} \nabla_{x} G\left(x_{i}, x_{j}\right)=0, \quad j=1, \ldots, m
$$

where $H(x, y)=G(x, y)-\frac{1}{2 \pi} \ln \frac{1}{|x-y|}$ is the regular part of $G(x, y)$. (See Brézis-Merle [4], Li-Shafrir [14], Li [13], Nagasaki-Suzuki [19], Ma-Wei [18].)

On the other hand, given $m$ points satisfying (3), Baraket-Pacard [2] constructed multiple bubbling solutions to $(2)$ when $h(x)=1$, and the bubble points satisfy nondegeneracy condition. Del Pino, Kowalczyk and Musso [8] constructed multiple bubbling solutions to (2) when the bubble points are topologically nontrivial. Furthermore, Chen-Lin [6, 7] obtained the sharp estimates for the bubbling rate of solutions to (2) as well as the LeraySchauder degree of all solutions to (2) for all $\rho \neq 8 m \pi$. A related question connected to physics consists in adding Dirac masses to the nonlinear parts, and we refer to Bartolucci-Chen-Lin-Tarantello [1] and to Tarantello [23] for backgrounds, results and asymptotics in this context. 
Going back to the Toda system (1), Jost-Wang [11] first classified the entire solutions. More precisely, for the following $\mathrm{SU}(3)$ system in $\mathbb{R}^{2}$

$$
\begin{cases}\Delta u+2 e^{u}-e^{v}=0 & \text { in } \mathbb{R}^{2} \\ \Delta v-e^{u}+2 e^{v}=0 & \text { in } \mathbb{R}^{2} \\ \int_{\mathbb{R}^{2}} e^{u}<\infty, \quad \int_{\mathbb{R}^{2}} e^{v}<\infty, & \end{cases}
$$

they showed that $(u, v)$ must be of the form:

$$
\begin{aligned}
& u(z)=\ln \frac{4\left(a_{1}^{2} a_{2}^{2}+a_{1}^{2}|2 z+c|^{2}+a_{2}^{2}\left|z^{2}+2 b z+b c-d\right|^{2}\right)}{\left(a_{1}^{2}+a_{2}^{2}|z+b|^{2}+\left|z^{2}+c z+d\right|^{2}\right)^{2}}, \\
& v(z)=\ln \frac{16 a_{1}^{2} a_{2}^{2}\left(a_{1}^{2}+a_{2}^{2}|z+b|^{2}+\left|z^{2}+c z+d\right|^{2}\right)}{\left(a_{1}^{2} a_{2}^{2}+a_{1}^{2}|2 z+c|^{2}+a_{2}^{2}\left|z^{2}+2 b z+b c-d\right|^{2}\right)^{2}},
\end{aligned}
$$

where $z=x_{1}+i x_{2} \in \mathbb{C}, a_{1}>0, a_{2}>0$ are real numbers and $b=b_{1}+i b_{2} \in \mathbb{C}$, $c=c_{1}+i c_{2} \in \mathbb{C}, d=d_{1}+i d_{2} \in \mathbb{C}$. Recently, Wei-Zhao-Zhou [24] obtained the nondegeneracy of the Jost-Wang's entire solution.

Jost-Wang [12] and Jost-Lin-Wang [10] studied the SU(3) Toda system on a two-dimensional manifold $M$ without boundary

$$
\begin{cases}\Delta u_{1 k}+2 \rho_{1 k} \frac{e^{u_{1 k}}}{\int_{M} e^{u_{1 k}}}-\rho_{2 k} \frac{e^{u_{2 k}}}{\int_{M} e^{u_{2 k}}}=0 & \text { on } M \\ \Delta u_{2 k}-\rho_{1 k} \frac{e^{u_{1 k}}}{\int_{M} e^{u_{1 k}}}+2 \rho_{2 k} \frac{e^{u_{2 k}}}{\int_{M} e^{u_{2 k}}}=0 & \text { on } M .\end{cases}
$$

They proved that the blow-up points of System (6) must be isolated. Furthermore, at each blow-up point, the limits of $\left(\rho_{1 k}, \rho_{2 k}\right)$ must be one of $(4 \pi, 0)$, $(0,4 \pi),(4 \pi, 8 \pi),(8 \pi, 4 \pi)$ and $(8 \pi, 8 \pi)$. See also related studies by OhtsukaSuzuki [20].

In this paper and the subsequent paper [17], we consider the blow-up analysis of solutions to (1). A first natural (and inevitable) question is whether or not there is a boundary bubble. Such a question will not arise in system (6). In the case of single equation (2), boundary blow-up is excluded by the method of moving planes and the use of Kelvin's transform ([18]). This technique works well for elliptic systems too, provided that the system is cooperative. More precisely, the elliptic system

$$
\Delta u+f(x, u, v)=0, \quad \Delta v+g(x, u, v)=0
$$

is cooperative if $\frac{\partial f(x, u, v)}{\partial v} \geq 0, \frac{\partial g(x, u, v)}{\partial u} \geq 0$. For the definition of cooperative systems and applications of the method of moving plane to cooperative systems, we refer to Troy [22] (for bounded domains) and Busca-Sirakov [5] (for 
the whole $\mathbb{R}^{N}$ ). See also Lin-Zhang [16] for the Liouville system which is cooperative. Unfortunately, the SU(3) Toda system is not cooperative because of the negative sign in the "off-diagonal" terms in (1). It is unclear if one can still use the method of moving planes. Instead, we shall use the Pohozaev identity and precise information on blow-ups to exclude boundary bubbles. This idea was introduced first by Robert-Wei [21] in studying the fourth order mean field equation with Dirichlet boundary conditions.

The purpose of this paper is to establish the corresponding properties (P1) and (P2) for System (1). Our main results can be stated as follows.

Theorem 1.1. Let $\left(u_{1 k}, u_{2 k}, \rho_{1 k}, \rho_{2 k}\right)$ be a sequence of solutions to (1) such that, as $k \rightarrow \infty$,

$$
0<\rho_{1 k} \leq \Lambda, 0<\rho_{2 k} \leq \Lambda \quad \text { and } \quad \max _{x \in \Omega} \max \left\{u_{1 k}, u_{2 k}\right\} \rightarrow+\infty .
$$

Then the blow-up set of $\max \left\{u_{1 k}, u_{2 k}\right\}$ is finite and in the interior of $\bar{\Omega}$. Precisely, there exists an $m \in \mathbb{N}^{*}$ (the set of positive integers) and a set $S=\left\{p_{1}, \ldots, p_{m}\right\} \subset \Omega$ such that (after taking a subsequence)

$$
u_{1 k} \rightarrow \sum_{i=1}^{m}\left(2 \sigma_{1 i}-\sigma_{2 i}\right) G\left(x, p_{i}\right), \quad u_{2 k} \rightarrow \sum_{i=1}^{m}\left(2 \sigma_{2 i}-\sigma_{1 i}\right) G\left(x, p_{i}\right)
$$

in $C_{\text {loc }}^{2}(\bar{\Omega} \backslash S)$, where $\left(\sigma_{1 i}, \sigma_{2 i}\right)$ can only be one of $(4 \pi, 0),(0,4 \pi),(4 \pi, 8 \pi)$, $(8 \pi, 4 \pi)$ or $(8 \pi, 8 \pi)$. Furthermore, $\rho_{1 k} \rightarrow \sum_{i=1}^{m} \sigma_{1 i}$ and $\rho_{2 k} \rightarrow \sum_{i=1}^{m} \sigma_{2 i}$.

This paper is organized as follows. In Section 2 we present some useful lemma and the Pohozaev identity. Theorem 1.1 is proved in Section 3.

Notation. Throughout this paper, we assume that $h_{1 k}, h_{2 k}$ are smooth positive functions converging to $h_{1}, h_{2}$ in $C^{2, \beta}(\bar{\Omega})$ respectively. The constant $C$ will denote various constants which are independent of $k$. All the convergence results are stated up to the extraction of a subsequence.

\section{Preliminary}

In this section we give some basic estimates and state the useful Pohozaev identity.

We first recall the following important estimate which can be found in [12, Theorem 3.1], [10, Proposition 2.1] and [10, Remark 2.1].

Theorem 2.1. Let $\Omega$ be a bounded smooth domain in $\mathbb{R}^{2}$ and $\left(u_{1 k}, u_{2 k}\right)$ be a sequence of solutions of the following system:

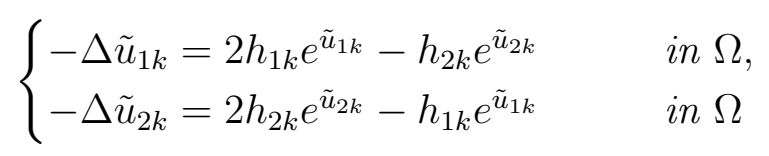


with

$$
\int_{\Omega} e^{\tilde{u}_{1 k}} \leq C \quad \text { and } \quad \int_{\Omega} e^{\tilde{u}_{2 k}} \leq C .
$$

Set

$$
S_{j}=\left\{x \in \Omega: \exists \text { a sequence } y^{k} \rightarrow x \text { such that } \tilde{u}_{j k}\left(y^{k}\right) \rightarrow+\infty\right\} .
$$

Then, one of the following possibilities happens (after taking subsequences):

(i) $\left(\tilde{u}_{1 k}, \tilde{u}_{2 k}\right)$ is uniformly bounded in $L_{l o c}^{\infty}(\Omega) \times L_{l o c}^{\infty}(\Omega)$.

(ii) For some $j \in\{1,2\}, u_{i k}$ is uniformly bounded in $L_{l o c}^{\infty}(\Omega)$, but $\tilde{u}_{j k} \rightarrow$ $-\infty$ uniformly in any compact subset of $\Omega$ for $j \neq i$.

(iii) For some $i \in\{1,2\}, S_{i} \neq \emptyset$ but $S_{j}=\emptyset$ for $j \neq i$. In this case, $\tilde{u}_{i k} \rightarrow-\infty$ uniformly in any compact subset of $\Omega \backslash S_{i}$, and either $\tilde{u}_{j k}$ is uniformly bounded in $L_{l o c}^{\infty}(\Omega)$ or $\tilde{u}_{j k} \rightarrow-\infty$ uniformly in any compact subset of $\Omega$.

(iv) $S_{1} \neq \emptyset$ and $S_{2} \neq \emptyset$, then both $\tilde{u}_{1 k}$ and $\tilde{u}_{2 k}$ tend to $-\infty$ uniformly in any compact subset of $\Omega \backslash\left\{S_{1} \cup S_{2}\right\}$.

Remark 2.2. The above theorem does not exclude the existence of boundary blow-up points. Actually, blow-up points lying on the boundary can exist in each one of the alternatives.

In what follows, we let

$$
\alpha_{1 k}=\ln \left(\frac{\int_{\Omega} h_{1 k} e^{u_{1 k}}}{\rho_{1 k}}\right), \quad \alpha_{2 k}=\ln \left(\frac{\int_{\Omega} h_{2 k} e^{u_{2 k}}}{\rho_{2 k}}\right)
$$

and

$$
\tilde{u}_{1 k}=u_{1 k}-\alpha_{1 k}, \quad \tilde{u}_{2 k}=u_{2 k}-\alpha_{2 k} .
$$

Then we have the following lemma.

Lemma 2.3. There exists a constant $C \in \mathbb{R}$ independent of $k$ such that $\alpha_{1 k} \geq C$ and $\alpha_{2 k} \geq C$ for all $k$.

Proof. Note that $\tilde{u}_{1 k}, \tilde{u}_{2 k}$ satisfy (8)-(9) and $\tilde{u}_{1 k}=-\alpha_{1 k}, \tilde{u}_{2 k}=-\alpha_{2 k}$ on $\partial \Omega$. Using Green's representation formula we have

$$
\begin{aligned}
& \tilde{u}_{1 k}(x)=\int_{\Omega} G(x, z)\left[2 h_{1 k}(z) e^{\tilde{u}_{1 k}(z)}-h_{2 k}(z) e^{\tilde{u}_{2 k}(z)}\right] \mathrm{d} z-\alpha_{1 k}, \\
& \tilde{u}_{2 k}(x)=\int_{\Omega} G(x, z)\left[2 h_{2 k}(z) e^{\tilde{u}_{2 k}(z)}-h_{1 k}(z) e^{\tilde{u}_{1 k}(z)}\right] \mathrm{d} z-\alpha_{2 k} .
\end{aligned}
$$


Thus we get

$$
\left\|\tilde{u}_{1 k}+\alpha_{1 k}\right\|_{L^{1}(\Omega)} \leq C, \quad\left\|\tilde{u}_{2 k}+\alpha_{2 k}\right\|_{L^{1}(\Omega)} \leq C .
$$

On the other hand, by Theorem 2.1, there exists an at most finite set $S_{1} \subset \Omega$ such that both $\tilde{u}_{1 k}$ and $\tilde{u}_{2 k}$ are uniformly bounded from above in any compact subset of $\Omega \backslash S_{1}$. Therefore, from (13) we see that $\alpha_{1 k}, \alpha_{2 k}$ cannot go to $-\infty$ as $k \rightarrow \infty$, which proves the lemma.

Lemma 2.4. Assume that $u_{1 k} \leq C$ and $u_{2 k} \leq C$. Then there exist $u_{1}, u_{2} \in$ $C^{2}(\bar{\Omega})$ such that (after taking a subsequence) $u_{1 k} \rightarrow u_{1}$ and $u_{2 k} \rightarrow u_{2}$ in $C^{2}(\bar{\Omega})$.

Proof. It follows from the assumption and Lemma 2.3 that $\tilde{u}_{1 k} \leq C$ and $\tilde{u}_{2 k} \leq C$ in $\Omega$. Thus we have that

$$
-\Delta u_{1 k} \in O(1), \quad-\Delta u_{2 k} \in O(1) .
$$

Elliptic regularity then implies the result.

We state a Pohozaev identity at the end of this section, which plays an important role in the proof of Theorem 1.1.

Lemma 2.5. It holds that, for any bounded domain $D \subset \mathbb{R}^{2}$,

$$
\begin{aligned}
\int_{D} 6 h_{1 k} e^{\tilde{u}_{1 k}}+\int_{D} 3 e^{\tilde{u}_{1 k}}\left\langle x-\xi, \nabla h_{1 k}\right\rangle \\
\quad+\int_{D} 6 h_{2 k} e^{\tilde{u}_{2 k}}+\int_{D} 3 e^{\tilde{u}_{2 k}}\left\langle x-\xi, \nabla h_{2 k}\right\rangle \\
=\int_{\partial D}\left(3 h_{1 k} e^{\tilde{u}_{1 k}}+3 h_{2 k} e^{\tilde{u}_{2 k}}\right)\langle x-\xi, \nu\rangle \\
\quad+\int_{\partial D} \frac{\partial\left(2 u_{1 k}+u_{2 k}\right)}{\partial \nu}\left\langle x-\xi, \nabla u_{1 k}\right\rangle+\int_{\partial D} \frac{\partial\left(u_{1 k}+2 u_{2 k}\right)}{\partial \nu}\left\langle x-\xi, \nabla u_{2 k}\right\rangle \\
\quad-\int_{\partial D}\left[\left|\nabla u_{1 k}\right|^{2}+\left\langle\nabla u_{1 k}, \nabla u_{2 k}\right\rangle+\left|\nabla u_{2 k}\right|^{2}\right]\langle x-\xi, \nu\rangle
\end{aligned}
$$

for any $\xi \in \mathbb{R}^{2}$.

Proof. We rewrite the system (1) as

$$
\left\{\begin{array}{l}
-\Delta\left(2 u_{1 k}+u_{2 k}\right)=3 h_{1 k} e^{\tilde{u}_{1 k}} \\
-\Delta\left(u_{1 k}+2 u_{2 k}\right)=3 h_{2 k} e^{\tilde{u}_{2 k}} .
\end{array}\right.
$$

Multiplying the first equation by $\left\langle x-\xi, \nabla u_{1 k}\right\rangle$, the second by $\left\langle x-\xi, \nabla u_{2 k}\right\rangle$, and integrating by parts, we obtain (14). 


\section{Proof of Theorem 1.1}

In this section, we give the proof of our main theorem 1.1.

Let

$$
M_{k}(x)=\max \left\{\tilde{u}_{1 k}(x), \tilde{u}_{2 k}(x)\right\},
$$

and $p_{k} \in \bar{\Omega}$ be such that

$$
M_{k}\left(p_{k}\right)=\max _{\bar{\Omega}} M_{k}(x)
$$

Define $\mu_{k}$ by

$$
-2 \ln \mu_{k}=M_{k}\left(p_{k}\right) .
$$

Note that $\mu_{k} \rightarrow 0$ by our assumption and hence $p_{k} \notin \partial \Omega$ since we know that $\left.M_{k}(x)\right|_{\partial \Omega} \leq C$ from Lemma 2.3 .

We prove first that the point $p_{k}$ must have some distance from the boundary.

Lemma 3.1. $\operatorname{dist}\left(p_{k}, \partial \Omega\right) / \mu_{k} \rightarrow \infty$.

Proof. Otherwise assume that there is a subsequence, still denoted by $\left(p_{k}, \mu_{k}\right)$, such that $\operatorname{dist}\left(p_{k}, \partial \Omega\right)=O\left(\mu_{k}\right)$. Let $\Omega_{k}=\left(\Omega-p_{k}\right) / \mu_{k}$. Then up to a rotation, we may assume that $\Omega_{k} \rightarrow\left(-\infty, t_{0}\right) \times \mathbb{R}$. With no loss of generality, we assume $\mu_{k}=e^{-\tilde{u}_{1 k}\left(p_{k}\right) / 2}$ and define

$$
\hat{u}_{1 k}(y)=\tilde{u}_{1 k}\left(p_{k}+\mu_{k} y\right)+2 \ln \mu_{k}+\ln h_{1 k}\left(p_{k}\right) .
$$

Let $R>0$ and $y \in B_{R}(0) \cap \Omega_{k}$. Then we have by the representation formula (13) that

$$
\begin{aligned}
\left|\nabla \hat{u}_{1 k}(y)\right| & =\left|\mu_{k} \nabla \tilde{u}_{1 k}\left(p_{k}+\mu_{k} y\right)\right| \\
& =\mu_{k}\left|\int_{\Omega} \nabla G\left(p_{k}+\mu_{k} y, z\right)\left[2 h_{1 k}(z) e^{\tilde{u}_{1 k}(z)}-h_{2 k}(z) e^{\tilde{u}_{2 k}(z)}\right] \mathrm{d} z\right| \\
& \leq C \mu_{k}\left[\int_{B_{2 \mu_{k}}\left(p_{k}\right)}+\int_{\Omega \backslash B_{2 R \mu_{k}}\left(p_{k}\right)}\right] \frac{\left|2 h_{1 k}(z) e^{\tilde{u}_{1 k}(z)}-h_{2 k}(z) e^{\tilde{u}_{2 k}(z)}\right|}{\left|p_{k}+\mu_{k} y-z\right|} \mathrm{d} z .
\end{aligned}
$$

Using the fact that $e^{\tilde{u}_{1 k}(z)}, e^{\tilde{u}_{2 k}(z)} \leq e^{\tilde{u}_{1 k}\left(p_{k}\right)}=\mu_{k}^{-2}$ in $B_{2 R \mu_{k}}\left(p_{k}\right)$, we know that

$$
\mu_{k} \int_{B_{2 R \mu_{k}}\left(p_{k}\right)} \frac{\left|2 h_{1 k}(z) e^{\tilde{u}_{1 k}(z)}-h_{2 k}(z) e^{\tilde{u}_{2 k}(z)}\right|}{\left|p_{k}+\mu_{k} y-z\right|} \mathrm{d} z \leq C(R) .
$$

Since $\mu_{k}\left|p_{k}+\mu_{k} y-z\right| \leq C(R)$ for $z \in \Omega \backslash B_{2 R \mu_{k}}\left(p_{k}\right)$, it is clear that 


$$
\begin{aligned}
\int_{\Omega \backslash B_{2 R \mu_{k}}\left(p_{k}\right)} \frac{\left|2 h_{1 k}(z) e^{\tilde{u}_{1 k}(z)}-h_{2 k}(z) e^{\tilde{u}_{2 k}(z)}\right|}{\left|p_{k}+\mu_{k} y-z\right|} \mathrm{d} z \\
\leq C(R) \int_{\Omega}\left|2 h_{1 k} e^{\tilde{u}_{1 k}}-h_{2 k} e^{\tilde{u}_{2 k}}\right| \leq C(R) .
\end{aligned}
$$

So we obtain that

$$
\left|\nabla \hat{u}_{1 k}\right| \leq C(R) \quad \text { in } B_{R}(0) \cap \Omega_{k},
$$

which particularly implies that $\left|\hat{u}_{1 k}(y)-\hat{u}_{1 k}(0)\right| \leq C|y| \leq C$ for any $y \in$ $\overline{B_{R}(0) \cap \Omega_{k}}$. For some fixed $y_{0} \in \partial \Omega_{k}$, we obtain that $\left|\hat{u}_{1 k}\left(y_{0}\right)-\hat{u}_{1 k}(0)\right|=$ $\left|\tilde{u}_{1 k}\left(p_{k}\right)+\alpha_{1 k}\right| \leq C$. This means that

$$
-2 \ln \mu_{k}+\alpha_{1 k}=O(1)
$$

which is a contradiction to Lemma 2.3 and the fact that $\mu_{k} \rightarrow 0$. Thus $\operatorname{dist}\left(p_{k}, \partial \Omega\right) / \mu_{k} \rightarrow \infty$.

For the function

$$
\begin{aligned}
& \hat{u}_{1 k}(y)=\tilde{u}_{1 k}\left(p_{k}+\mu_{k} y\right)+2 \ln \mu_{k}+\ln h_{1 k}\left(p_{k}\right), \\
& \hat{u}_{2 k}(y)=\tilde{u}_{2 k}\left(p_{k}+\mu_{k} y\right)+2 \ln \mu_{k}+\ln h_{2 k}\left(p_{k}\right),
\end{aligned}
$$

we have the following lemma.

Lemma 3.2. In any compact subset of $\mathbb{R}^{2}$, (after taking a subsequence) either (a) $\left(\hat{u}_{1 k}, \hat{u}_{2 k}\right)$ converges to one of the Jost-Wang's entire solution (4), (5); or

(b) one of $\hat{u}_{1 k}, \hat{u}_{2 k}$ converges to a solution of Liouville equation

$$
\Delta u+e^{u}=0 \text { in } \mathbb{R}^{2}, \quad \int_{\mathbb{R}^{2}} e^{u}<\infty
$$

and the other converges to $-\infty$ uniformly on compact subsets of $\mathbb{R}^{2}$.

Proof. By Lemma 3.1 we have $\Omega_{k}=\left(\Omega-p_{k}\right) / \mu_{k} \rightarrow \mathbb{R}^{2}$. It is easy to check that

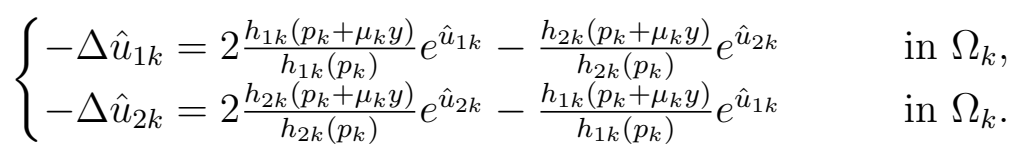

We can verify that $\hat{u}_{1 k}$ and $\hat{u}_{2 k}$ satisfy the conditions of Theorem 2.1. Since by definition $\hat{u}_{1 k} \leq C$ and $\hat{u}_{2 k} \leq C$, there are two possibilities (after taking a subsequence):

1) $\hat{u}_{1 k}$ and $\hat{u}_{2 k}$ are both uniformly locally bounded in $\mathbb{R}^{2}$; 
2) either $\hat{u}_{1 k}$ or $\hat{u}_{2 k}$ is uniformly locally bounded in $\mathbb{R}^{2}$, while the other one diverges to $-\infty$ uniformly on compact subset of $\mathbb{R}^{2}$.

For Case 1$)$, one can show that $\left(\hat{u}_{1 k}, \hat{u}_{2 k}\right)$ converges in $C_{\text {loc }}^{2}\left(\mathbb{R}^{2}\right)$ to an entire solution with finite energy. Now the classification result [11] gives $(a)$.

For Case 2), let $\hat{u}_{1 k}$ be uniformly locally bounded, then it is easy to check that $\hat{u}_{1 k}$ converges to a solution of the Liouville equation (3.2).

The proof is concluded.

To establish the finiteness of the blow-up points set, we introduce a definition. We say that the property $\mathcal{H}_{\boldsymbol{\ell}}$ holds if there exists $\left(p_{k, 1}, \ldots, p_{k, \ell}\right)$ such that, denoting $\mu_{k, j}=e^{-\max \left\{\tilde{u}_{1 k}\left(p_{k, j}\right), \tilde{u}_{2 k}\left(p_{k, j}\right)\right\} / 2} \rightarrow 0$, we have that

i) $\lim _{k \rightarrow \infty}\left|p_{k, i}-p_{k, j}\right| / \mu_{k, j}=\infty$ for any $i \neq j$;

ii) $\lim _{k \rightarrow \infty} \operatorname{dist}\left(p_{k, i}, \partial \Omega\right) / \mu_{k, i}=\infty$ for all $i=1, \ldots, \ell$;

iii) for all $i=1, \ldots, \ell$, we denote $\hat{u}_{1 k, i}(y)=\tilde{u}_{1 k}\left(p_{k, i}+\mu_{k, i} y\right)+2 \ln \mu_{k, i}+$ $\ln h_{1 k}\left(p_{k, i}\right)$ and $\hat{u}_{2 k, i}(y)=\tilde{u}_{2 k}\left(p_{k, i}+\mu_{k, i} y\right)+2 \ln \mu_{k, i}+\ln h_{2 k}\left(p_{k, i}\right)$, then in any compact subset of $\mathbb{R}^{2}$, either $\left(\hat{u}_{1 k, i}, \hat{u}_{2 k, i}\right)$ converges to the JostWang's entire solution of Toda system; or one of $\hat{u}_{1 k, i}, \hat{u}_{2 k, i}$ converges to a solution of Liouville equation (3.2) and the other diverges to $-\infty$ on compact subsets of $\mathbb{R}^{2}$.

We remark that Lemma 3.1 and Lemma 3.2 imply that $\mathcal{H}_{1}$ holds. From $i i i$ ) and Fatou's lemma, we deduce that if $\mathcal{H}_{\ell}$ holds, then for every $i=1, \ldots, \ell$,

$$
\lim _{r \rightarrow 0} \lim _{k \rightarrow \infty} \int_{B_{r}\left(p_{k, i}\right)} h_{1 k} e^{\tilde{u}_{1 k}}+\lim _{r \rightarrow 0} \lim _{k \rightarrow \infty} \int_{B_{r}\left(p_{k, i}\right)} h_{2 k} e^{\tilde{u}_{2 k}} \geq 4 \pi .
$$

Lemma 3.3. Assume that $\mathcal{H}_{\ell}$ holds. Then either $\mathcal{H}_{\ell+1}$ holds, or there exists $C>0$ such that

$$
\inf _{i=1, \ldots, \ell}\left|x-p_{k, i}\right|^{2} e^{\max \left\{\tilde{u}_{1 k}(x), \tilde{u}_{2 k}(x)\right\}} \leq C .
$$

Proof. Let $w_{k}(x)=\inf _{i=1, \ldots, \ell}\left|x-p_{k, i}\right|^{2} e^{M_{k}(x)}$ where $M_{k}(x)$ is defined in (15). Assume that $\left\|w_{k}\right\|_{L^{\infty}(\Omega)} \rightarrow \infty$. Note that $\left.M_{k}(x)\right|_{\partial \Omega} \leq C$. Now let $x_{k} \in \Omega$ be such that $w_{k}\left(x_{k}\right)=\max _{\bar{\Omega}} w_{k}$ and $\gamma_{k}=e^{-M_{k}\left(x_{k}\right) / 2}$. Observe that $\gamma_{k} \rightarrow 0$ and $w_{k}\left(x_{k}\right)=\inf _{i=1, \ldots, \ell}\left|x_{k}-p_{k, i}\right|^{2} / \gamma_{k}^{2} \rightarrow \infty$. Thus

$$
\frac{\left|x_{k}-p_{k, i}\right|}{\gamma_{k}} \rightarrow \infty \quad \text { for all } i=1, \ldots, \ell .
$$


Assume that there exists some $j$ such that $x_{k}-p_{k, j}=O\left(\mu_{k, j}\right)$. Then we may write $x_{k}=p_{k, j}+\mu_{k, j} \theta_{k, j}$ where $\theta_{k, j}=O(1)$. Because of the property (iii), direct computations show that

$$
\left|x_{k}-p_{k, j}\right|^{2} e^{M_{k}\left(x_{k}\right)}=\left|\theta_{k, j}\right|^{2} e^{M_{k}\left(p_{k, j}+\mu_{k, j} \theta_{k, j}\right)+2 \ln \mu_{k, j}} \rightarrow C\left(\theta_{\infty, j}\right)<+\infty,
$$

which is a contradiction to the fact that $w_{k}\left(x_{k}\right) \rightarrow \infty$. Thus we have

$$
\frac{\left|x_{k}-p_{k, i}\right|}{\mu_{k, i}} \rightarrow \infty \quad \text { for all } i=1, \ldots, \ell .
$$

Let $y \in B_{R}(0) \cap \Omega_{k}$ where $\Omega_{k}=\left(\Omega-x_{k}\right) / \gamma_{k}$ and let $\epsilon \in(0,1)$. Then $w_{k}\left(x_{k}+\gamma_{k} y\right) \leq w_{k}\left(x_{k}\right)$. That is, $\inf _{i=1, \ldots, \ell}\left|x_{k}+\gamma_{k} y-p_{k, i}\right| e^{M_{k}\left(x_{k}+\gamma_{k} y\right)} \leq$ $\inf _{i=1, \ldots, \ell}\left|x_{k}-p_{k, i}\right| e^{M_{k}\left(x_{k}\right)}$. We now define $v_{i k}(y)=\tilde{u}_{i k}\left(x_{k}+\gamma_{k} y\right)+2 \ln \gamma_{k}+$ $\ln h_{i k}\left(x_{k}\right)(i=1,2)$. Then it is easy to check that

$$
e^{\max \left\{v_{1 k}(y), v_{2 k}(y)\right\}} \leq \frac{Q_{1} \inf _{i=1, \ldots, \ell}\left|x_{k}-p_{k, i}\right|^{2}}{\inf _{i=1, \ldots, \ell}\left|x_{k}+\gamma_{k} y-p_{k, i}\right|^{2}},
$$

where the constant $Q_{1}>0$ is chosen to satisfy that $Q_{1} \geq \max _{x \in \bar{\Omega}}\left\{h_{1 k}(x), h_{2 k}(x)\right\}$ because of the uniform boundedness of $h_{1 k}$ and $h_{2 k}$. Let $k(R)$ be such that $\left|x_{k}-p_{k, i}\right| / \gamma_{k} \geq \frac{R}{\epsilon}$ for all $i=1, \ldots, \ell$ and $k \geq k(R)$ in view of (17). Thus for all $i$ we have $\left|x_{k}+\gamma_{k} y-p_{k, i}\right| \geq\left|x_{k}-p_{k, i}\right|(1-\epsilon)$, which yields that

$$
\begin{aligned}
\max \left\{v_{1 k}(y), v_{2 k}(y)\right\} & \leq \ln \frac{Q_{1}}{(1-\epsilon)^{2}} \quad \text { for any } y \in B_{R}(0) \cap \Omega_{k}, k \geq k(R), \\
e^{M_{k}\left(x_{k}+\gamma_{k} y\right)} & \leq \frac{Q_{1}}{Q_{2}(1-\epsilon)^{2}} \gamma_{k}^{-2} \quad \text { for any } y \in B_{R}(0) \cap \Omega_{k}, k \geq k(R),
\end{aligned}
$$

where the constant $Q_{2}>0$ is chosen to satisfy that $Q_{2} \leq \min _{x \in \bar{\Omega}}\left\{h_{1 k}(x), h_{2 k}(x)\right\}$. Substituting $\hat{u}_{1 k}, \hat{u}_{2 k}, \mu_{k}$ by $v_{1 k}, v_{2 k}, \gamma_{k}$ in the proof of Lemma 3.1, we obtain that

$$
\frac{\operatorname{dist}\left(x_{k}, \partial \Omega\right)}{\gamma_{k}} \rightarrow \infty
$$

Similarly, Lemma 3.2 also holds for $\left(v_{1 k}, v_{2 k}\right)$. Let $p_{k, \ell+1}=x_{k}, \mu_{k, \ell+1}=\gamma_{k}$. Combining (17), (18) and the above remark, we conclude that $\mathcal{H}_{\ell+1}$ holds.

Lemma 3.4. There exists some $m \in \mathbb{N}^{*}$ such that $\mathcal{H}_{m}$ holds and

$$
\inf _{i=1, \ldots, m}\left|x-p_{k, i}\right|^{2} e^{\max \left\{\tilde{u}_{1 k}(x), \tilde{u}_{2 k}(x)\right\}} \leq C \quad \text { for any } x \in \Omega .
$$


Proof. Suppose not. Since $\mathcal{H}_{1}$ holds, $\mathcal{H}_{\ell}$ holds for all $\ell \geq 1$ by the above lemma. From the property $(i)$, given $R>0$, we have $B_{R \mu_{k, i}}\left(p_{k, i}\right) \cap B_{R \mu_{k, j}}\left(p_{k, j}\right)=$ $\emptyset$ for all $i \neq j, k \geq k(R)$. Recall the assumption that $\rho_{1 k}, \rho_{2 k} \leq \Lambda$. By (16), it is easy to check that

$$
\begin{aligned}
2 \Lambda & \geq \lim _{k \rightarrow \infty}\left(\rho_{1 k}+\rho_{2 k}\right)=\lim _{k \rightarrow \infty} \int_{\Omega} h_{1 k} e^{\tilde{u}_{1 k}}+\lim _{k \rightarrow \infty} \int_{\Omega} h_{2 k} e^{\tilde{u}_{2 k}} \\
& \geq \lim _{k \rightarrow \infty} \sum_{i=1}^{\ell} \int_{B_{R \mu_{k, i}}\left(p_{k, i}\right)} h_{1 k} e^{\tilde{u}_{1 k}}+h_{2 k} e^{\tilde{u}_{2 k}} \\
& \geq 4 \pi \ell,
\end{aligned}
$$

which implies that $\ell \leq \Lambda /(2 \pi)$ and leads to a contradiction. The proof is complete.

Lemma 3.5. There exists a $C>0$ such that

$$
\inf _{i=1, \ldots, m}\left|x-p_{k, i}\right|\left|\nabla \tilde{u}_{1 k}(x)\right| \leq C, \quad \inf _{i=1, \ldots, m}\left|x-p_{k, i}\right|\left|\nabla \tilde{u}_{2 k}(x)\right| \leq C,
$$

for all $x \in \Omega$.

Proof. By Green's representation formula (11), we have

$$
\left|\nabla \tilde{u}_{1 k}\right| \leq C \int_{\Omega} \frac{1}{|x-z|}\left[2 h_{1 k}(z) e^{\tilde{u}_{1 k}(z)}-h_{2 k}(z) e^{\tilde{u}_{2 k}(z)}\right] \mathrm{d} z .
$$

Let $R_{k}(x)=\inf _{i=1, \ldots, m}\left|x-p_{k, i}\right|$ and $\Omega_{k, i}=\left\{x \in \Omega:\left|x-p_{k, i}\right|=R_{k}(x)\right\}$ for $i=1, \ldots, m$. Note that $\Omega=\bigcup_{i=1}^{m} \Omega_{k, i}$ for each $k$. Then for $x \in \Omega$,

$$
\begin{aligned}
& \int_{\Omega_{k, i}} \frac{h_{1 k}(z) e^{\tilde{u}_{1 k}(z)}}{|x-z|} \mathrm{d} z=\int_{\Omega_{k, i} \cap B_{\left|x-p_{k, i}\right| / 2}\left(p_{k, i}\right)} \frac{h_{1 k}(z) e^{\tilde{u}_{1 k}(z)}}{|x-z|} \mathrm{d} z \\
&+\int_{\Omega_{k, i} \backslash B_{\left|x-p_{k, i}\right| / 2}\left(p_{k, i}\right)} \frac{h_{1 k}(z) e^{\tilde{u}_{1 k}(z)}}{|x-z|} \mathrm{d} z .
\end{aligned}
$$

Using (19), we note that for $z \in \Omega_{k, i} \backslash B_{\left|x-p_{k, i}\right| / 2}\left(p_{k, i}\right)$,

$$
\frac{h_{1 k}(z) e^{\tilde{u}_{1 k}(z)}}{|x-z|} \leq \frac{C}{|x-z|\left|z-p_{k, i}\right|^{2}} \leq \frac{C}{|x-z|\left|x-p_{k, i}\right|^{2}} .
$$

Simple computation then shows that

$$
\int_{\Omega_{k, i} \backslash B_{\left|x-p_{k, i}\right| / 2}\left(p_{k, i}\right)} \frac{h_{1 k}(z) e^{\tilde{u}_{1 k}(z)}}{|x-z|} \mathrm{d} z \leq \frac{C}{\left|x-p_{k, i}\right|} .
$$


On the other hand, for $z \in \Omega_{k, i} \cap B_{\left|x-p_{k, i}\right| / 2}\left(p_{k, i}\right)$, we have $|x-z| \geq \frac{1}{2}\left|x-p_{k, i}\right|$ and hence

$$
\int_{\Omega_{k, i} \cap B_{\left|x-p_{k, i}\right| / 2}\left(p_{k, i}\right)} \frac{h_{1 k}(z) e^{\tilde{u}_{1 k}(z)}}{|x-z|} \mathrm{d} z \leq \frac{C}{\left|x-p_{k, i}\right|} .
$$

By (21)-(23), it holds that

$$
\int_{\Omega_{k, i}} \frac{h_{1 k}(z) e^{\tilde{u}_{1 k}(z)}}{|x-z|} \mathrm{d} z \leq \frac{C}{\left|x-p_{k, i}\right|} .
$$

Similarly, it also holds that

$$
\int_{\Omega_{k, i}} \frac{h_{2 k}(z) e^{\tilde{u}_{2 k}(z)}}{|x-z|} \mathrm{d} z \leq \frac{C}{\left|x-p_{k, i}\right|} .
$$

Finally we obtain that, from (20),

$$
\inf _{i=1, \ldots, m}\left|x-p_{k, i}\right|\left|\nabla \tilde{u}_{1 k}(x)\right| \leq C .
$$

The estimate for $\nabla \tilde{u}_{2 k}$ may be proved analogously. The proof is finished.

Denote $p_{i}=\lim _{k \rightarrow \infty} p_{k, i} \in \bar{\Omega}$ for all $i=1, \ldots, m$ and $S=\left\{p_{1}, \ldots, p_{m}\right\}$.

Lemma 3.6. $u_{1 k}$ and $u_{2 k}$ are uniformly bounded in any compact subset of $\bar{\Omega} \backslash S$.

Proof. We just prove the result for $u_{1 k}$. The proof is similar for $u_{2 k}$. Let $\delta>0$ be small enough such that $\Omega_{\delta}=\bar{\Omega} \backslash \bigcup_{i=1}^{m} B_{\delta}\left(p_{i}\right)$ is connected. So we have $\inf _{i=1, \ldots, m}\left|x-p_{k, i}\right| \geq \frac{\delta}{2}$ for all $x \in \Omega_{\delta}$ as long as $k$ large enough. Thus we get from Lemma 3.5 that $\left|\nabla \tilde{u}_{1 k}\right| \leq C(\delta)$. Then $\left|\nabla u_{1 k}\right|=\left|\nabla \tilde{u}_{1 k}\right| \leq C(\delta)$ in $\Omega_{\delta}$. Thus for some $x_{\delta} \in \partial \Omega_{\delta} \cap \partial \Omega$, we have

$$
\left|u_{1 k}(x)\right|=\left|u_{1 k}(x)-u_{1 k}\left(x_{\delta}\right)\right| \leq C(\delta)
$$

for all $x \in \Omega_{\delta}$. The proof is completed.

Remark 3.7. The blow-up set of $\max \left\{u_{1 k}, u_{2 k}\right\}$ is exactly $S$. In fact, Lemma 3.6 says that it must be contained in $S$. On the other hand, since $S$ is the blow-up set of $\max \left\{\tilde{u}_{1 k}, \tilde{u}_{2 k}\right\}$ and $\alpha_{1 k}, \alpha_{2 k} \geq C, \max \left\{u_{1 k}, u_{2 k}\right\}$ also blows up at $S$. 
Lemma 3.8. Assume that one of $\alpha_{1 k}, \alpha_{2 k}$ is uniformly bounded. Then $S \subset \partial \Omega$. Moreover, assume that $\alpha_{1 k} \rightarrow \alpha_{1 \infty}$ and $\alpha_{2 k} \rightarrow \alpha_{2 \infty}$ (up to a subsequence), then there exists $u_{1 \infty}, u_{2 \infty} \in C^{2}(\bar{\Omega})$ such that

$$
\begin{cases}-\Delta u_{1 \infty}=2 h_{1} e^{u_{1 \infty}-\alpha_{1 \infty}}-h_{2} e^{u_{2 \infty}-\alpha_{2 \infty}} & \text { in } \Omega, \\ -\Delta u_{2 \infty}=2 h_{2} e^{u_{2 \infty}-\alpha_{2 \infty}}-h_{1} e^{u_{1 \infty}-\alpha_{1 \infty}} & \text { in } \Omega, \\ u_{1 \infty}=u_{2 \infty}=0 & \text { on } \partial \Omega\end{cases}
$$

and

$$
u_{1 k} \rightarrow u_{1 \infty}, \quad u_{2 k} \rightarrow u_{2 \infty} \quad \text { in } C_{l o c}^{2}(\bar{\Omega} \backslash S) .
$$

(Here $e^{u_{i \infty}-\alpha_{i \infty}}=0$ if $\alpha_{i \infty}=+\infty(i=1,2)$.)

Proof. First we prove that $S \subset \partial \Omega$. Note that there exist two possibilities:

1) $\alpha_{1 k}, \alpha_{2 k}$ are both uniformly bounded;

2) (up to a subsequence) $\alpha_{1 k} \rightarrow+\infty, \alpha_{2 k} \rightarrow \alpha_{2 \infty}<+\infty$ or $\alpha_{1 k} \rightarrow \alpha_{1 \infty}<$ $+\infty, \alpha_{2 k} \rightarrow+\infty$.

For Case 1), from (13) it holds that $\left\|\tilde{u}_{1_{k}}\right\|_{L^{1}(\Omega)} \leq C$ and $\left\|\tilde{u}_{2 k}\right\|_{L^{1}(\Omega)} \leq C$. According to Theorem 2.1, we have that $\tilde{u}_{1 k}$ and $\tilde{u}_{2 k}$ must be both uniformly bounded in $L_{\text {loc }}^{\infty}(\Omega)$. Since $S$ is the blow-up set, we conclude that $S \subset \partial \Omega$.

For Case 2), without loss of generality, we assume that $\alpha_{1 k} \rightarrow+\infty$, $\alpha_{2 k} \rightarrow \alpha_{2 \infty}<+\infty$. Since $\left\|\tilde{u}_{2 k}\right\|_{L^{1}(\Omega)} \leq C$, by Theorem 2.1, we know that $\tilde{u}_{2 k}$ is uniformly bounded in $L_{\text {loc }}^{\infty}(\Omega)$. Lemma 3.6 and the fact that $\left|\alpha_{2 k}\right| \leq C$ further imply that

both $u_{2 k}$ and $\tilde{u}_{2 k}$ are uniformly bounded in $L_{\text {loc }}^{\infty}(\bar{\Omega} \backslash(S \cap \partial \Omega))$.

Thus standard elliptic theory implies that $u_{1 k}+2 u_{2 k}$ is uniformly bounded in $C_{\text {loc }}^{1}(\bar{\Omega} \backslash(S \cap \partial \Omega))$. Therefore $u_{1 k}$ is uniformly bounded in $L_{\text {loc }}^{\infty}(\bar{\Omega} \backslash(S \cap \partial \Omega))$ and, because $\alpha_{1 k} \rightarrow+\infty$,

$$
\tilde{u}_{1 k} \rightarrow-\infty \text { uniformly in any compact subset of } \bar{\Omega} \backslash(S \cap \partial \Omega) \text {. }
$$

Since $S$ is the blow-up set, we again conclude that $S \subset \partial \Omega$ by (25) and (26).

It follows from Lemma 3.4 and standard elliptic theory that there exist $u_{1 \infty}, u_{2 \infty} \in C^{2}(\bar{\Omega} \backslash S)$ such that

$$
u_{1 k} \rightarrow u_{1 \infty}, \quad u_{2 k} \rightarrow u_{2 \infty} \quad \text { in } C_{\mathrm{loc}}^{2}(\bar{\Omega} \backslash S) .
$$

Thus, passing to the limit $k \rightarrow \infty$ in Lemma 3.5, we get that

$$
\inf _{i=1, \ldots, m}\left|x-p_{i}\right|\left|\nabla u_{1 \infty}(x)\right| \leq C \quad \text { for all } x \in \bar{\Omega} \backslash S,
$$




$$
\inf _{i=1, \ldots, m}\left|x-p_{i}\right|\left|\nabla u_{2 \infty}(x)\right| \leq C \quad \text { for all } x \in \bar{\Omega} \backslash S .
$$

It remains to prove that $u_{1 \infty}, u_{2 \infty}$ can be smoothly extended to $S$. We fix some $p_{j} \in S \subset \partial \Omega$ and let $\delta>0$ small enough such that

$$
\left|x-p_{j}\right|\left|\nabla u_{1 \infty}(x)\right| \leq C \quad \forall x \in \bar{\Omega} \cap B_{\delta}\left(p_{j}\right) \backslash\left\{p_{j}\right\} .
$$

Therefore, there exists $C^{\prime}>0$ such that for all $x, z \in \bar{\Omega} \cap B_{\delta}\left(p_{j}\right) \backslash\left\{p_{j}\right\}$ such that $\left|x-p_{j}\right|=\left|z-p_{j}\right|$, we have that

$$
\left|u_{1 \infty}(x)-u_{1 \infty}(z)\right| \leq C^{\prime}
$$

Taking $z \in \partial \Omega \cap B_{\delta}\left(p_{j}\right) \backslash\left\{p_{j}\right\}$, we then get $\left|u_{1 \infty}(x)\right| \leq C^{\prime}$ for all $x \in$ $\bar{\Omega} \cap B_{\delta}\left(p_{j}\right) \backslash\left\{p_{j}\right\}$. Recalling that $S \subset \partial \Omega$ and taking the similar procedure for all the points of $S$, we get that there exists $C>0$ such that $\left|u_{1 \infty}(x)\right| \leq C$ for all $x \in \bar{\Omega} \backslash S$. Similarly we also obtain that $\left|u_{2 \infty}(x)\right| \leq C$ for all $x \in \bar{\Omega} \backslash S$.

Let $w \in H_{0}^{1}(\Omega)$ such that $-\Delta w=2 h_{1 \infty} e^{u_{1 \infty}-\alpha_{1 \infty}}-h_{2 \infty} e^{u_{2 \infty}-\alpha_{2 \infty}}$ in $\Omega$. It follows from standard elliptic theory that $w \in C^{1}(\bar{\Omega})$ and

$$
w(x)=\int_{\Omega} G(x, z)\left[2 h_{1 \infty}(z) e^{u_{1 \infty}(z)-\alpha_{1 \infty}}-h_{2 \infty}(z) e^{u_{2 \infty}(z)-\alpha_{2 \infty}}\right] \mathrm{d} z .
$$

For any fixed $x \in \bar{\Omega} \backslash S$ and any fixed $\delta>0$ small enough,

$$
\begin{aligned}
u_{1 k}(x)= & \int_{\Omega} G(x, z)\left[2 h_{1 k}(z) e^{\tilde{u}_{1 k}(z)}-h_{2 k}(z) e^{\tilde{u}_{2 k}(z)}\right] \mathrm{d} z \\
= & \int_{\Omega \backslash \bigcup_{i=1}^{m} B_{\delta}\left(p_{i}\right)} G(x, z)\left[2 h_{1 k}(z) e^{\tilde{u}_{1 k}(z)}-h_{2 k}(z) e^{\tilde{u}_{2 k}(z)}\right] \mathrm{d} z \\
& \quad+\int_{\bigcup_{i=1}^{m} B_{\delta}\left(p_{i}\right) \cap \Omega} G(x, z)\left[2 h_{1 k}(z) e^{\tilde{u}_{1 k}(z)}-h_{2 k}(z) e^{\tilde{u}_{2 k}(z)}\right] \mathrm{d} z .
\end{aligned}
$$

Note that $G\left(x, p_{i}\right)=0$ for $p_{i} \in \partial \Omega$ and $x \neq p_{i}$. Using (27) and passing to the limit (first in $k$ and then in $\delta$ ) in the above equality, we achieve that

$$
u_{1 \infty}(x)=\int_{\Omega} G(x, z)\left[2 h_{1 \infty}(z) e^{\tilde{u}_{1 \infty}(z)}-h_{2 \infty}(z) e^{\tilde{u}_{2 \infty}(z)}\right] \mathrm{d} z,
$$

which means that $u_{1 \infty} \equiv w$ in $\bar{\Omega} \backslash S$ and therefore $u_{1 \infty}$ can be extended as a $C^{1}(\bar{\Omega})$ function. Coming back to the equation of $w$, we get that $w$ is $C^{2}(\bar{\Omega})$ and then $u_{1 \infty} \in C^{2}(\bar{\Omega})$.

Similar procedure may be applied to $u_{2 \infty}$. The proof is accomplished. 
Lemma 3.9. We have that (up to a subsequence)

$$
\alpha_{1 k} \rightarrow+\infty \quad \text { and } \quad \alpha_{2 k} \rightarrow+\infty
$$

Proof. Otherwise we know that $S \subset \partial \Omega$ by Lemma 3.8. Choose $x_{0} \in S$ and $r>0$ small enough such that $S \cap B_{r}\left(x_{0}\right)=\left\{x_{0}\right\}$. Let $z_{k}=x_{0}+\vartheta_{k, r} \nu\left(x_{0}\right)$ with

$$
\vartheta_{k, r}=\frac{\int_{\partial \Omega \cap B_{r}\left(x_{0}\right)}\left\langle x-x_{0}, \nu\right\rangle\left[\left|\frac{\partial u_{1 k}}{\partial \nu}\right|^{2}+\frac{\partial u_{1 k}}{\partial \nu} \frac{\partial u_{2 k}}{\partial \nu}+\left|\frac{\partial u_{2 k}}{\partial \nu}\right|^{2}\right]}{\int_{\partial \Omega \cap B_{r}\left(x_{0}\right)}\left\langle\nu\left(x_{0}\right), \nu\right\rangle\left[\left|\frac{\partial u_{1 k}}{\partial \nu}\right|^{2}+\frac{\partial u_{1 k}}{\partial \nu} \frac{\partial u_{2 k}}{\partial \nu}+\left|\frac{\partial u_{2 k}}{\partial \nu}\right|^{2}\right]}
$$

where $r$ is small such that $\frac{1}{2} \leq\left\langle\nu\left(x_{0}\right), \nu\right\rangle \leq 1$ for $x \in \partial \Omega \cap B_{r}\left(x_{0}\right)$. Here $\nu(x)$ is the unit outer normal at $x \in \partial \Omega$. It is then easy to check that $\left|\vartheta_{k, r}\right| \leq 2 r$ for $\left|\left\langle x-x_{0}, \nu\right\rangle\right| \leq r$. Observing $x-z_{k}=x-x_{0}-\vartheta_{k, r} \nu\left(x_{0}\right)$, we know that

$$
\int_{\partial \Omega \cap B_{r}\left(x_{0}\right)}\left\langle x-z_{k}, \nu\right\rangle\left[\left|\frac{\partial u_{1 k}}{\partial \nu}\right|^{2}+\frac{\partial u_{1 k}}{\partial \nu} \frac{\partial u_{2 k}}{\partial \nu}+\left|\frac{\partial u_{2 k}}{\partial \nu}\right|^{2}\right]=0 .
$$

Now applying Pohozaev identity (14) in $\Omega \cap B_{r}\left(x_{0}\right)$ with $\xi=z_{k}$, we have that

$$
\begin{aligned}
& \int_{\Omega \cap B_{r}\left(x_{0}\right)} 6 h_{1 k} e^{u_{1 k}-\alpha_{1 k}}+\int_{\Omega \cap B_{r}\left(x_{0}\right)} 3 e^{u_{1 k}-\alpha_{1 k}}\left\langle x-z_{k}, \nabla h_{1 k}\right\rangle \\
& \quad+\int_{\Omega \cap B_{r}\left(x_{0}\right)} 6 h_{2 k} e^{u_{2 k}-\alpha_{2 k}}+\int_{\Omega \cap B_{r}\left(x_{0}\right)} 3 e^{u_{2 k}-\alpha_{2 k}}\left\langle x-z_{k}, \nabla h_{2 k}\right\rangle \\
& =\int_{\partial\left(\Omega \cap B_{r}\left(x_{0}\right)\right)}\left(3 h_{1 k} e^{u_{1 k}-\alpha_{1 k}}+3 h_{2 k} e^{u_{2 k}-\alpha_{2 k}}\right)\left\langle x-z_{k}, \nu\right\rangle \\
& \quad+\int_{\partial\left(\Omega \cap B_{r}\left(x_{0}\right)\right)} \frac{\partial\left(2 u_{1 k}+u_{2 k}\right)}{\partial \nu}\left\langle x-z_{k}, \nabla u_{1 k}\right\rangle+\frac{\partial\left(u_{1 k}+2 u_{2 k}\right)}{\partial \nu}\left\langle x-z_{k}, \nabla u_{2 k}\right\rangle \\
& \quad-\int_{\partial\left(\Omega \cap B_{r}\left(x_{0}\right)\right)}\left[\left|\nabla u_{1 k}\right|^{2}+\left\langle\nabla u_{1 k}, \nabla u_{2 k}\right\rangle+\left|\nabla u_{2 k}\right|^{2}\right]\left\langle x-z_{k}, \nu\right\rangle .
\end{aligned}
$$

In view of the boundary conditions and Lemma 2.3, it is easy to see that

$$
\lim _{k \rightarrow \infty} \int_{\partial \Omega \cap B_{r}\left(x_{0}\right)}\left(3 h_{1 k} e^{u_{1 k}-\alpha_{1 k}}+3 h_{2 k} e^{u_{2 k}-\alpha_{2 k}}\right)\left\langle x-z_{k}, \nu\right\rangle=O\left(r^{2}\right)
$$

and, by (28),

$$
\begin{gathered}
\int_{\partial \Omega \cap B_{r}\left(x_{0}\right)} \frac{\partial\left(2 u_{1 k}+u_{2 k}\right)}{\partial \nu}\left\langle x-z_{k}, \nabla u_{1 k}\right\rangle+\frac{\partial\left(u_{1 k}+2 u_{2 k}\right)}{\partial \nu}\left\langle x-z_{k}, \nabla u_{2 k}\right\rangle \\
-\int_{\partial \Omega \cap B_{r}\left(x_{0}\right)}\left[\left|\nabla u_{1 k}\right|^{2}+\left\langle\nabla u_{1 k}, \nabla u_{2 k}\right\rangle+\left|\nabla u_{2 k}\right|^{2}\right]\left\langle x-z_{k}, \nu\right\rangle
\end{gathered}
$$




$$
=\int_{\partial \Omega \cap B_{r}\left(x_{0}\right)}\left\langle x-z_{k}, \nu\right\rangle\left[\left|\frac{\partial u_{1 k}}{\partial \nu}\right|^{2}+\frac{\partial u_{1 k}}{\partial \nu} \frac{\partial u_{2 k}}{\partial \nu}+\left|\frac{\partial u_{2 k}}{\partial \nu}\right|^{2}\right]=0 .
$$

From (24) of Lemma 3.8, we have $\left\|u_{1 k}\right\|_{C^{2}\left(\bar{\Omega} \cap \partial B_{r}\left(x_{0}\right)\right)} \leq C$ (independent of $r$ ). (Similar estimates hold for $u_{2 k}$.) We obtain that

$$
\lim _{k \rightarrow \infty} \int_{\Omega \cap \partial B_{r}\left(x_{0}\right)}\left(3 h_{1 k} e^{u_{1 k}-\alpha_{1 k}}+3 h_{2 k} e^{u_{2 k}-\alpha_{2 k}}\right)\left\langle x-z_{k}, \nu\right\rangle=O\left(r^{2}\right)
$$

and

$$
\begin{aligned}
& \lim _{k \rightarrow \infty} \int_{\Omega \cap \partial B_{r}\left(x_{0}\right)} \frac{\partial\left(2 u_{1 k}+u_{2 k}\right)}{\partial \nu}\left\langle x-z_{k}, \nabla u_{1 k}\right\rangle+\frac{\partial\left(u_{1 k}+2 u_{2 k}\right)}{\partial \nu}\left\langle x-z_{k}, \nabla u_{2 k}\right\rangle \\
& \quad-\lim _{k \rightarrow \infty} \int_{\Omega \cap \partial B_{r}\left(x_{0}\right)}\left[\left|\nabla u_{1 k}\right|^{2}+\left\langle\nabla u_{1 k}, \nabla u_{2 k}\right\rangle+\left|\nabla u_{2 k}\right|^{2}\right]\left\langle x-z_{k}, \nu\right\rangle \\
= & O\left(r^{2}\right) .
\end{aligned}
$$

Since $\int_{\Omega} h_{i k} e^{\tilde{u}_{i k}} \leq C$, it holds that

$$
\lim _{k \rightarrow \infty} \int_{\Omega \cap B_{r}\left(x_{0}\right)} 3 e^{u_{i k}-\alpha_{i k}}\left\langle x-z_{k}, \nabla h_{i k}\right\rangle=O(r) \quad i=1,2 .
$$

Then we have, by taking the limit to (29) first in $k$ and then in $r$,

$$
\lim _{r \rightarrow 0} \lim _{k \rightarrow \infty} \int_{\Omega \cap B_{r}\left(x_{0}\right)} h_{1 k} e^{u_{1 k}-\alpha_{1 k}}+\lim _{r \rightarrow 0} \lim _{k \rightarrow \infty} \int_{\Omega \cap B_{r}\left(x_{0}\right)} h_{2 k} e^{u_{2 k}-\alpha_{2 k}}=0,
$$

which is a contradiction to (16).

Lemma 3.10. There exist $\left(\sigma_{1 i}, \sigma_{2 i}\right)$ satisfying $\sigma_{1 i}+\sigma_{2 i} \geq 4 \pi(i=1, \ldots, m)$ such that (up to a subsequence)

$$
\begin{array}{ll}
u_{1 k}(x) \rightarrow \sum_{i=1}^{m}\left(2 \sigma_{1 i}-\sigma_{2 i}\right) G\left(x, p_{i}\right) & \text { in } C_{l o c}^{2}(\bar{\Omega} \backslash S), \\
u_{2 k}(x) \rightarrow \sum_{i=1}^{m}\left(2 \sigma_{2 i}-\sigma_{1 i}\right) G\left(x, p_{i}\right) & \text { in } C_{l o c}^{2}(\bar{\Omega} \backslash S) .
\end{array}
$$

Proof. Note that $\tilde{u}_{1 k}, \tilde{u}_{2 k} \rightarrow-\infty$ uniformly in any compact subset of $\bar{\Omega} \backslash S$. For any fixed $x \in \bar{\Omega} \backslash S$ and any fixed $\delta>0$ small enough,

$$
\lim _{k \rightarrow \infty} u_{1 k}(x)=\lim _{k \rightarrow \infty} \int_{\Omega} G(x, z)\left[2 h_{1 k}(z) e^{\tilde{u}_{1 k}(z)}-h_{2 k}(z) e^{\tilde{u}_{2 k}(z)}\right] \mathrm{d} z
$$




$$
=\lim _{k \rightarrow \infty} \sum_{i=1}^{m} \int_{B_{\delta}\left(p_{i}\right) \cap \Omega} G(x, z)\left[2 h_{1 k}(z) e^{\tilde{u}_{1 k}(z)}-h_{2 k}(z) e^{\tilde{u}_{2 k}(z)}\right] \mathrm{d} z .
$$

Since $G(x, \cdot)$ is continuous in $\bar{\Omega} \backslash\{x\}$, we pass the limit in $\delta \rightarrow 0$ and get that

$$
\lim _{k \rightarrow \infty} u_{1 k}(x)=\sum_{i=1}^{m}\left(2 \sigma_{1 i}-\sigma_{2 i}\right) G\left(x, p_{i}\right)
$$

where $\sigma_{1 i}=\lim _{\delta \rightarrow 0} \lim _{k \rightarrow \infty} \int_{B_{\delta}\left(p_{i}\right) \cap \Omega} h_{1 k} e^{\tilde{u}_{1 k}}$ and $\sigma_{2 i}=\lim _{\delta \rightarrow 0} \lim _{k \rightarrow \infty} \int_{B_{\delta}\left(p_{i}\right) \cap \Omega} h_{2 k} e^{\tilde{u}_{2 k}}$ and $\sigma_{1 i}+\sigma_{2 i} \geq 4 \pi$ from (16). Similarly, we also have

$$
\lim _{k \rightarrow \infty} u_{2 k}(x)=\sum_{i=1}^{m}\left(2 \sigma_{2 i}-\sigma_{1 i}\right) G\left(x, p_{i}\right)
$$

Finally, standard elliptic theory shows that the convergence is of $C_{\text {loc }}^{2}(\bar{\Omega} \backslash$ $S)$.

Lemma 3.11. $S \cap \partial \Omega=\emptyset$.

Proof. We argue by contradiction. Let $x_{0} \in S \cap \partial \Omega$. We may assume further that $S \cap B_{\delta}\left(x_{0}\right)=\left\{x_{0}\right\}$. Arguing as in Lemma 3.9, we get that

$$
\begin{aligned}
& \int_{\Omega \cap B_{r}\left(x_{0}\right)} 6 h_{1 k} e^{u_{1 k}-\alpha_{1 k}}+\int_{\Omega \cap B_{r}\left(x_{0}\right)} 3 e^{u_{1 k}-\alpha_{1 k}}\left\langle x-z_{k}, \nabla h_{1 k}\right\rangle \\
& \quad+\int_{\Omega \cap B_{r}\left(x_{0}\right)} 6 h_{2 k} e^{u_{2 k}-\alpha_{2 k}}+\int_{\Omega \cap B_{r}\left(x_{0}\right)} 3 e^{u_{2 k}-\alpha_{2 k}}\left\langle x-z_{k}, \nabla h_{2 k}\right\rangle \\
& =\int_{\partial\left(\Omega \cap B_{r}\left(x_{0}\right)\right)}\left(3 h_{1 k} e^{u_{1 k}-\alpha_{1 k}}+3 h_{2 k} e^{u_{2 k}-\alpha_{2 k}}\right)\left\langle x-z_{k}, \nu\right\rangle \\
& \quad+\int_{\partial\left(\Omega \cap B_{r}\left(x_{0}\right)\right)} \frac{\partial\left(2 u_{1 k}+u_{2 k}\right)}{\partial \nu}\left\langle x-z_{k}, \nabla u_{1 k}\right\rangle+\frac{\partial\left(u_{1 k}+2 u_{2 k}\right)}{\partial \nu}\left\langle x-z_{k}, \nabla u_{2 k}\right\rangle \\
& \quad-\int_{\partial\left(\Omega \cap B_{r}\left(x_{0}\right)\right)}^{\left[\left|\nabla u_{1 k}\right|^{2}+\left\langle\nabla u_{1 k}, \nabla u_{2 k}\right\rangle+\left|\nabla u_{2 k}\right|^{2}\right]\left\langle x-z_{k}, \nu\right\rangle .}
\end{aligned}
$$

Using Lemma 3.10 and noting that $G\left(x, x_{0}\right)=0$ for any $x \in \Omega \cap \partial B_{r}\left(x_{0}\right)$, we obtain that

$$
\lim _{k \rightarrow \infty} \int_{\Omega \cap \partial B_{r}\left(x_{0}\right)}\left(3 h_{1 k} e^{u_{1 k}-\alpha_{1 k}}+3 h_{2 k} e^{u_{2 k}-\alpha_{2 k}}\right)\left\langle x-z_{k}, \nu\right\rangle=O\left(r^{2}\right),
$$

and 


$$
\begin{aligned}
& \lim _{k \rightarrow \infty} \int_{\Omega \cap \partial B_{r}\left(x_{0}\right)} \frac{\partial\left(2 u_{1 k}+u_{2 k}\right)}{\partial \nu}\left\langle x-z_{k}, \nabla u_{1 k}\right\rangle+\frac{\partial\left(u_{1 k}+2 u_{2 k}\right)}{\partial \nu}\left\langle x-z_{k}, \nabla u_{2 k}\right\rangle \\
& -\lim _{k \rightarrow \infty} \int_{\Omega \cap \partial B_{r}\left(x_{0}\right)}\left[\left|\nabla u_{1 k}\right|^{2}+\left\langle\nabla u_{1 k}, \nabla u_{2 k}\right\rangle+\left|\nabla u_{2 k}\right|^{2}\right]\left\langle x-z_{k}, \nu\right\rangle=O\left(r^{2}\right) .
\end{aligned}
$$

This implies then

$$
\lim _{r \rightarrow 0} \lim _{k \rightarrow \infty} \int_{\Omega \cap B_{r}\left(x_{0}\right)} 6 h_{1 k} e^{u_{1 k}-\alpha_{1 k}}+\lim _{r \rightarrow 0} \lim _{k \rightarrow \infty} \int_{\Omega \cap B_{r}\left(x_{0}\right)} 6 h_{2 k} e^{u_{2 k}-\alpha_{2 k}}=0,
$$

which is a contradiction.

So far we have proved that $S \subset \Omega$ and $\alpha_{1 k} \rightarrow+\infty$ and $\alpha_{2 k} \rightarrow+\infty$. Thus Proposition 2.4 of [10] shows that $\left(\sigma_{1 i}, \sigma_{2 i}\right)$ of Lemma 3.10 can only be one of $(4 \pi, 0),(0,4 \pi),(4 \pi, 8 \pi),(8 \pi, 4 \pi)$ or $(8 \pi, 8 \pi)$. Finally, since $\tilde{u}_{1 k} \rightarrow-\infty$ and $\tilde{u}_{2 k} \rightarrow-\infty$ locally in $\bar{\Omega} \backslash S$,

$$
\lim _{k \rightarrow \infty} \rho_{1 k}=\lim _{k \rightarrow \infty} \int_{\Omega} h_{1 k} e^{\tilde{u}_{1 k}}=\lim _{r \rightarrow 0} \lim _{k \rightarrow \infty} \sum_{i=1}^{m} \int_{B_{r}\left(p_{i}\right)} h_{1 k} e^{\tilde{u}_{1 k}}=\sum_{i=1}^{m} \sigma_{1 i} .
$$

Similarly we have $\rho_{2 k} \rightarrow \sum_{i=1}^{m} \sigma_{2 i}$. This completes the proof of Theorem 1.1.

\section{ACKNOWLEDGMENTS}

The research of Wei is partially supported by a Competitive Earmarked Grant from RGC of Hong Kong. Wei and Zhao's research is also partially supported by a Focused Research Scheme from CUHK. This work was carried out while the third author was visiting the Taida Institute of Mathematical Sciences: he thanks this institution for its support and its hospitality. Finally we thank the referee for providing detailed suggestions which greatly improve the presentation of the paper.

\section{References}

[1] D. Bartolucci, C.-C. Chen, C.-S. Lin, G. Tarantello, Profile of blow-up solutions to mean field equations with singular data, Comm. Partial Differential Equations, 29, (2004), 1241-1265.

[2] S. Baraket and F. Pacard, Construction of singular limits for a semilinear elliptic equation in dimension 2, Calc. Var. Partial Differential Equations, 6, (1998), 1-38. 
[3] H. Brezis, Y.Y. Li, I. Shafrir, A sup + inf inequality for some nonlinear elliptic equations involving exponential nonlinearities. J. Funct. Anal. 115 (1993), no. 2, 344-358.

[4] H. Brezis, F. Merle, Uniform estimates and blow-up behavior for solutions of $-\Delta u=V(x) e^{u}$ in two dimensions. Comm. Partial Differential Equations 16 (1991), no. 8-9, 1223-1253.

[5] J. Busca, B. Sirakov, Symmetry results for semilinear elliptic systems in the whole space. J. Differential Equations 163 (2000), no. 1, 41C56.

[6] C.-C. Chen and C.-S. Lin, Sharp estimates for solutions of multi-bubbles in compact Riemann surface, Comm. Pure Appl. Math., 55, (2002), 728771.

[7] C.-C. Chen and C.-S. Lin, Topological degree for a mean field equation on Riemann surfaces, Comm. Pure Appl. Math., 56, (2003), 1667-1727.

[8] M. Del Pino, M. Kowalczyk, M. Musso, Singular limits in Liouville-type equations. Calc. Var. Partial Differential Equations, 24, (2005), 47-81.

[9] G. Dunne, Self-Dual Chern-Simons theories, Lecture notes in physics: Monographs 36, Springer-Verlag, Berlin Heidelberg, 1995.

[10] J. Jost, C.-S. Lin, G.F. Wang, Analytic aspects of the Toda system. II. Bubbling behavior and existence of solutions. Comm. Pure Appl. Math. 59 (2006), no. 4, 526-558.

[11] J. Jost, G.F. Wang, Classification of solutions of a Toda system in $\mathbb{R}^{2}$. Int. Math. Res. Not. 2002, no. 6, 277-290.

[12] J. Jost, G.F. Wang, Analytic aspects of the Toda system: I. A MoserTrudinger inequality. Comm. Pure Appl. Math. 54 (2001), no. 11, 12891319 .

[13] Y.Y. Li, Harnack type inequality: the method of moving planes. Comm. Math. Phys. 200 (1999), no. 2, 421-444.

[14] Y.Y. Li, I. Shafrir, Blow-up analysis for solutions of $-\Delta u=V e^{u}$ in dimension two. Indiana Univ. Math. J. 43 (1994), no. 4, 1255-1270.

[15] C.-S. Lin, J.C. Wei, Sharp estimates for bubbling solutions of a fourth order mean field equation. Ann. Sc. Norm. Super. Pisa Cl. Sci. (5) 6 (2007), no. 4, 599-630. 
[16] C.-S. Lin, L. Zhang, Profile of bubbling solutions to a Liouville system. Ann. Inst. H. Poincaré Anal. Non Linéaire 27 (2010), no. 1, 117-143.

[17] C.-S. Lin, J.C. Wei, C.Y. Zhao, Refined asymptotic behavior of SU(3) Toda system in a bounded domain, preprint.

[18] L. Ma, J.C. Wei, Convergence for a Liouville equation. Comment. Math. Helv. 76 (2001), no. 3, 506-514.

[19] K. Nagasaki, T. Suzuki, Asymptotic analysis for two-dimensional elliptic eigenvalue problems with exponentially dominated nonlinearities. Asymptotic Anal. 3 (1990), no. 2, 173-188.

[20] H. Ohtsuka and T. Suzuki, Blow-up analysis for SU(3) Toda system, J. Differential Equations 232(2007), no.2, 419-440.

[21] F. Robert, J.C. Wei, Asymptotic behavior of a fourth order mean field equation with Dirichlet boundary condition. Indiana Univ. Math. J. 57 (2008), no. 5, 2039-2060.

[22] W. Troy, Symmetry properties in systems of semilinear elliptic equations, J. Differential Equations 42 (1981), 400-413.

[23] G. Tarantello, Selfdual gauge field vortices. An analytical approach. Progress in Nonlinear Differential Equations and their Applications, 72. Birkhäuser Boston, Inc., Boston, MA, 2008. xiv+325 pp. ISBN: 978-08176-4310-2.

[24] J.C. Wei, C.Y. Zhao, F. Zhou, On nondegeneracy of solutions to SU(3) Toda system. C.R.A.S., to appear.

[25] Y.S. Yang, Solitons in field theory and nonlinear analysis. Springer Monographs in Mathematics. Springer, New York, 2001. 